\title{
Novel Injectable Pentablock Copolymer Based Thermoresponsive Hydrogels for Sustained Release Vaccines
}

\author{
Sharan Bobbala, ${ }^{1}$ Viral Tamboli, ${ }^{2}$ Arlene McDowell, ${ }^{1}$ Ashim K. Mitra, ${ }^{2}$ and Sarah Hook ${ }^{1,3}$
}

Received 18 August 2015; accepted 22 October 2015; published online 20 November 2015

\begin{abstract}
The need for multiple vaccinations to enhance the immunogenicity of subunit vaccines may be reduced by delivering the vaccine over an extended period of time. Here, we report two novel injectable pentablock copolymer based thermoresponsive hydrogels made of polyethyleneglycol-polycaprolactonepolylactide-polycaprolactone-polyethyleneglycol (PEG-PCL-PLA-PCL-PEG) with varying ratios of polycaprolactone (PCL) and polylactide (PLA), as single shot sustained release vaccines. Pentablock copolymer hydrogels were loaded with vaccine-encapsulated poly lactic-co-glycolic acid nanoparticles (PLGA-NP) or with the soluble vaccine components. Incorporation of PLGA-NP into the thermoresponsive hydrogels increased the complex viscosity of the gels, lowered the gelation temperature, and minimized the burst release of antigen and adjuvants. The two pentablock hydrogels stimulated both cellular and humoral responses. The addition of PLGA-NP to the hydrogels sustained immune responses for up to 49 days. The polymer with a higher ratio of PCL to PLA formed a more rigid gel, induced stronger immune responses, and stimulated effective anti-tumor responses in a prophylactic melanoma tumor model.
\end{abstract}

KEY WORDS: nanoparticles; pentablock copolymers; sustained release; thermoresponsive hydrogels; vaccine delivery.

\section{INTRODUCTION}

Subunit vaccines that utilize defined peptide, protein, or nucleic acid antigens are less reactogenic than wholepathogen vaccines (1). However, use of subunit vaccines also results in poor immunogenicity due to lack of secondary signals (2). The weak immunogenicity of subunit vaccines may be overcome by delivering the vaccine multiple times or through using formulation strategies such as incorporating the antigen into sustained release formulations and including immune stimulatory adjuvants in the vaccine. The release of antigen in a sustained manner may increase the period of antigen interaction with the immune cells and thus lead to the induction of effective immune responses $(3,4)$. Care must be taken with the development of such formulations as inappropriate release of vaccine antigen and adjuvant can induce cell sequestration and deletion (5).

Delivering subunit vaccines via particulate carriers to mimic pathogens is a strategy to enhance the immunogenicity of subunit vaccines (6). Additionally, particulate carriers may

Electronic supplementary material The online version of this article (doi:10.1208/s12248-015-9843-4) contains supplementary material, which is available to authorized users.

\footnotetext{
${ }^{1}$ School of Pharmacy, University of Otago, Dunedin, 9054, New Zealand.

${ }^{2}$ School of Pharmacy, UMKC, Kansas City, Missouri, USA.

${ }^{3}$ To whom correspondence should be addressed. (e-mail: sarah.hook@otago.ac.nz)
}

facilitate the synchronous presentation of vaccine antigens and adjuvants to dendritic cells, decreasing the potential for the development of tolerogenic immune response (7). Poly lactic-co-glycolic acid nanoparticles (PLGA-NP) have been extensively studied for vaccine delivery and have been reported to target dendritic cells naturally through phagocytosis with efficient delivery of the vaccine components (8). PLGA-NP can accommodate a wide range of actives (9) and in the present study were loaded with the model vaccine antigen ovalbumin and the adjuvants monophosphoryl lipid A (MPL) and Quil A (QA) before being combined with thermoresponsive hydrogels with the aim of creating a oneshot vaccine. MPL is a non-toxic, Toll-like receptor (TLR) 4 ligand isolated from the gram negative bacterium, Salmonella minnesota (10). MPL was the first TLR ligand to be approved for commercial use in humans and is reported to induce strong Th1 immune responses (11). Quil A is a non-TLRdependent saponin adjuvant extracted from the bark of Quillaja saponaria (12). QA is capable of stimulating both cytotoxic T cell and humoral responses (13). Both MPL and QA can stimulate improved immune responses when administered individually, and recent studies suggest a combination of these adjuvants can induce stronger immune responses compared to each adjuvant delivered separately $(14,15)$.

Thermoresponsive or in situ-forming hydrogels are temperature-sensitive injectable systems that are polymeric solutions at ambient temperature and that rapidly transform into sustained release gel depots upon administration into the body (16). Actives such as drugs and vaccines can be 
incorporated into hydrogels by simply mixing with the temperature-sensitive polymer solutions at ambient temperature (17). The triblock copolymer poloxamer 407 has been examined extensively as an in situ gelling system but the fast erosion of these gels in aqueous environments and the nonbiodegradability of the polymer has limited its use as a sustained release system $(18,19)$. Attempts to increase the stability of poloxamer through the addition of copolymers such as pluronic 25R4 (20), dextran (21), carrageenan (22), and methylcellulose (23) have had limited success. Biodegradable triblock copolymers made up of polycaprolactone (PCL) and polyethyleneglycol (PEG) (i.e., PEG-PCL-PEG, PCL-PEG-PCL) are reported to be stable in aqueous conditions but the crystallinity of the PCL blocks slowed degradation (24-26). In this study, two novel thermosensitive pentablock copolymers consisting of polyethyleneglycolpolycaprolactone-polylactide-polycaprolactonepolyethyleneglycol (PEG-PCL-PLA-PCL-PEG) were studied as injectable hydrogels, with the polylactide (PLA) blocks being added to accelerate the degradation of PCL $(27,28)$. These tailor-made pentablock copolymers were developed for the sustained ocular delivery of drugs (29).

The overall aim of the current study was to develop a one-shot sustained release vaccine. Initial work in this study focussed on the preparation of pentablock copolymer hydrogels containing soluble and nanoparticle vaccine formulations. This was followed by rheological analysis, in vitro stability, and release studies to investigate the behavior of thermoresponsive hydrogels as sustained release formulations with and without PLGA-NP. Further in vivo vaccine and challenge studies were performed to examine the potential of these formulations in generating cellular and humoral responses.

\section{MATERIALS AND METHODS}

\section{Materials}

Poly(ethylene glycol), (PEG: 1 and $4 \mathrm{kDa}$ ), methoxyPEG (550 Da), stannous octoate, $\varepsilon$-caprolactone, albumin from chicken egg (OVA, grade V, purity approximately $98 \%$ ), fluorescein isothiocyanate (FITC, isomer 1), monophosphoryl lipid A from S. minnesota RE 595, and formic acid were acquired from Sigma-Aldrich (Missouri, USA). L-lactide and hexamethylenediamine (HMDI) were purchased from Acros organics (Morris Plains, NJ, USA). Purified saponin (Quil$A ®)$ was obtained from Brenntag Biosector (Frederikssund, Denmark). PLGA copolymer with a 50:50 monomer ratio and inherent viscosity of 0.55 to $0.75 \mathrm{dL} / \mathrm{g}$ was acquired from Absorbable Polymers International (Pelham, USA). Polyvinyl alcohol (PVA), MW 25,000, was purchased from Polysciences, Inc. (Warrington, USA). Poloxamer 407 (Lutrol® F127) and Pluronic ${ }^{\circledR}$ 25R4 were obtained from BASF (Ludwigshafen, Germany). HPLC-grade chloroform, methanol, and acetonitrile were purchased form Merck KGaA (Darmstadt, Germany). Alum was obtained from SERVA Electrophoresis (Heidelberg, Germany). All the antibodies used for flow cytometry analysis were obtained from BD Pharmingen (San Diego, CA, USA). Water was purified using a Millipore ${ }^{\circledR}$ Purification System (USA) and was used during sample preparation and HPLC analysis.
Nitrogen gas (oxygen free) was supplied by BOC Ltd., (Auckland, NZ).

\section{Preparation of PLGA-NP}

PLGA-NP were prepared by the double-emulsion method as previously reported $(30,31)$. In the primary emulsion step, FITC-OVA (prepared as previously described (32)) or OVA $(50 \mu \mathrm{L}, 25 \mathrm{mg} / \mathrm{mL}$ in PBS $)$ and QA $(50 \mu \mathrm{L}, 50 \mathrm{mg} / \mathrm{mL}$ in PBS) were added to the PLGA $(300 \mu \mathrm{L}, 10 \% w / v$ in chloroform) and MPL (100 $\mu \mathrm{L}, 25 \mathrm{mg} / \mathrm{mL}$ in chloroform). The resultant mixture was emulsified using an ultrasonic processor UP50H (Teltow, Germany) to form a primary emulsion. This primary emulsion was added to $2 \mathrm{~mL}$ of PVA solution ( $6 \% w / v$ PVA) and further emulsified by sonication to form a secondary emulsion. The secondary emulsion was then added drop wise into $8 \mathrm{~mL}$ of stirring $0.5 \%$ PVA solution at room temperature. Nanoparticles were collected after $3 \mathrm{~h}$ of stirring followed by centrifugation at $10,000 \times \mathrm{g}$ (Eppendorf centrifuge, Hamburg, Germany) for $10 \mathrm{~min}$. After centrifugation, nanoparticles were washed twice with cold water to remove the residual PVA and redispersed in PBS.

Particle-size distribution and zeta potential of PLGA-NP were determined by photon correlation spectroscopy (PCS) and electrophoretic mobility, using a Zetasizer Nano-ZS (Malvern Instruments, UK).

\section{Synthesis, Preparation, and Loading of Thermosensitive Hydrogels}

The two pentablock copolymers $\mathrm{PEG}_{550^{-}} \mathrm{PCL}_{825}-\mathrm{PLA}_{550^{-}}$ $\mathrm{PCL}_{825}-\mathrm{PEG}_{550}(\mathrm{P} 1)$ and $\mathrm{PEG}_{550}-\mathrm{PCL}_{550}-\mathrm{PLA}_{1100}-\mathrm{PCL}_{550^{-}}$ $\mathrm{PEG}_{550}(\mathrm{P} 2)$ with different ratios of PCL and PLA blocks were synthesized as reported previously (29). Solutions (17\% $w / v)$ of the two pentablock copolymers in PBS ( $\mathrm{pH} 7.4)$ were stirred overnight at $4^{\circ} \mathrm{C}$. A triblock copolymer hydrogel (Tri), consisting of Poloxamer 407 and pluronic 25R4, was prepared as described previously (20). PLGA-NP containing OVA (or FITC-OVA when required), MPL and QA or soluble OVA (or FITC-OVA when required), MPL and QA were added to the thermosensitive polymer solutions which were then stirred overnight at $4^{\circ} \mathrm{C}$ to ensure homogenous mixing. All formulations contained $100 \mu \mathrm{g} / \mathrm{mL}$ OVA (or FITC-OVA when required), $100 \mu \mathrm{g} / \mathrm{mL}$ MPL, and $200 \mu \mathrm{g} / \mathrm{mL}$ QA.

\section{Entrapment of FITC-OVA, MPL, and QA in PLGA-NP}

An aliquot $(20 \mathrm{mg})$ of PLGA-NP was added to $2 \mathrm{~mL}$ of $0.5 \mathrm{M}$ sodium hydroxide and incubated overnight in dark with stirring to ensure complete dissolution of particles. Entrapment of FITC-OVA was determined by fluorescence spectroscopy (Shimadzu RF-540, Shimadzu Corporation, Kyoto, Japan) at excitation and emission wavelengths of 490 and $520 \mathrm{~nm}$, respectively. The fluorescence of standard solutions of sodium hydroxide treated FITC-OVA was used to calculate the amount of FITC-OVA entrapped within PLGA-NP.

The loading of MPL and QA in PLGA-NP was quantified by a high-performance liquid chromatography/ evaporative light scattering detection (HPLC-ELSD) method as previously described (33). 


\section{Rheological Measurements}

Rheology measurements were performed using a cone and plate geometry on an Oscillatory HR-3 rheometer (TA Instruments). The rheometer was equipped with a temperature-controlled peltier plate and a $60-\mathrm{mm}$ cone with an angle of $2^{\circ}$. All the measurements were performed in the linear viscoelastic region of the polymer. The sol-gel transition temperature was determined using temperature sweep measurements with $1^{\circ} \mathrm{C}$ intervals from 10 to $40^{\circ} \mathrm{C}$. The sol-gel transition time and the angular frequency sweep measurements were determined at $37^{\circ} \mathrm{C}$. All the samples were placed on the peltier plate at $5^{\circ} \mathrm{C}$ and a solvent trap was used to prevent evaporation of the sample.

\section{In Vitro Gel Stability Studies}

One milliliter of thermosensitive polymer solution was transferred into a vial and incubated at $37^{\circ} \mathrm{C}$ to induce gelation. The initial weight of the gel was noted (W1) and $1 \mathrm{~mL}$ of PBS prewarmed to $37^{\circ} \mathrm{C}$ was carefully layered on the gel surface. At predetermined time intervals, the PBS was removed, the weight of the remaining gel was recorded (W2), and the percentage gel weight loss was calculated.

\section{Fluorescence Microscopy}

FITC-OVA-loaded PLGA-NP, thermosensitive hydrogels containing soluble FITC-OVA, and FITC-OVA PLGA-NP were analyzed by fluorescence microscopy using an IX71/IX81 Olympus microscope (Olympus America, Inc.).

\section{In Vitro Release Studies}

In vitro release of FITC-OVA, MPL, and QA from PLGA-NP, PLGA-NP in hydrogels, and hydrogels was determined. A 20-mg aliquot of PLGA nanoparticles was transferred into Eppendorf tubes, and $1 \mathrm{~mL}$ of PBS was added. The samples were incubated at $37^{\circ} \mathrm{C}$ in the dark, and at predetermined time intervals the amount of FITC-OVA, QA, and MPL remaining in the nanoparticles was determined as described above.

A 1-mL aliquot of the thermosensitive polymer solutions containing vaccine-loaded PLGA-NP or soluble FITC-OVA, $\mathrm{QA}$, and MPL was transferred into vials and incubated at $37^{\circ} \mathrm{C}$ to induce gelation. One milliliter of PBS prewarmed to $37^{\circ} \mathrm{C}$ was carefully layered over the surface of gels, and the vials were incubated at $37^{\circ} \mathrm{C}$ in the dark. At predetermined time intervals, $100 \mu \mathrm{L}$ of release media was removed and replaced with an equal volume of PBS. FITC-OVA, QA, and MPL levels in the release media were then analyzed as described above and expressed as cumulative amount released (percent) versus time.

\section{Mice}

Male C57Bl/6 and OT-I and OT-II transgenic mice, 68 weeks old were bred and housed under specific pathogenfree conditions at the HTRU, Dunedin, New Zealand. T cell receptors specific for the CD8 $\left(\mathrm{OVA}_{257-264}\right)$ and $\mathrm{CD} 4$ (OVA $323-339)$ peptide epitopes of OVA are highly expressed in OT-I and OT-II transgenic mice, respectively $(34,35)$. All the mice had free access to food and water during the entire period of experiments. All experiments were approved by the University of Otago Animal Ethics Committee.

\section{Adoptive T Cell Transfer}

OT-I and OT-II transgenic cells were transferred into $\mathrm{C} 57 \mathrm{Bl} / 6$ mice on the day before immunization (4). In brief, spleens and lymph nodes were collected from OT-I and OT-II transgenic mice, a single-cell suspension at $1 \times 10^{6}$ cells $/ \mathrm{mL}$ was prepared, and $200 \mu \mathrm{L}$ was injected into the tail vein of $\mathrm{C} 57 \mathrm{Bl} / 6$ mice the day before immunization.

\section{Immunization Protocol}

All the mice were injected with $200 \mu \mathrm{L}$ of vaccine formulation subcutaneously in the dorsal skin fold on day 0 of experiment. Formulations used were OVA-MPL-QA entrapped PLGA-NP, thermosensitive polymer solutions loaded with OVA-MPL-QA entrapped PLGA-NP, and soluble OVA, MPL, and QA in thermosensitive polymer solutions. Each animal received a total of $20 \mu \mathrm{g}$ of OVA, $20 \mu \mathrm{g}$ of MPL, and $40 \mu \mathrm{g}$ of QA (36). Alum containing $20 \mu \mathrm{g}$ of OVA was given as the control formulation. Three days prior to sampling, all the mice were injected with $10 \mu \mathrm{g}$ of OVA to maximize the detected immune response. Mice were sacrificed 21, 30, or 49 days after immunization and draining (axial and brachial) lymph nodes and blood were collected for further analysis.

\section{Measurement of Cellular and Humoral Immune Responses}

Single-cell suspensions were prepared from harvested lymph nodes, stained and analyzed by flow cytometry (BD FACS Canto II, BD Biosciences). Cells were first incubated with anti-CD16/CD32 antibody (2.4G2) and subsequently stained with an antibody mix consisting of anti-CD8 (53-6.7FITC), anti-CD4 (APC), anti-V 22 (B20.1-PE), and antiVB5.1 (MR9-4-biotin). Secondary staining with SA-APCCy7 was then carried out. Data was analyzed using FlowJo software version 10.0.6 (Tree Star, Inc.) and representative flow cytometry plots are shown in supplementary Fig. 1. The total number of CD8 or CD4 transgenic cells was calculated by multiplying the percentage of antigen specific $\mathrm{V} \alpha 2^{+} \mathrm{V} \beta 5^{+}$ CD8 T cells or $\mathrm{V} \alpha 2^{+} \mathrm{V} \beta 5^{+} \mathrm{CD} 4 \mathrm{~T}$ cells by the total number of viable lymphocytes (37).

Serum was collected from centrifuged $(2700 \times g$ for $10 \mathrm{~min}$ ) blood samples and stored at $-20^{\circ} \mathrm{C}$ till analysis. The serum samples were analyzed by ELISA for OVA-specific IgG antibody (38).

\section{Tumor Experiments}

Mice (eight per group) received either no treatment or were immunized subcutaneously in the dorsal skin fold with P1 hydrogels with blank PLGA-NP, P1 hydrogels with soluble vaccine, or P1 hydrogels with PLGA-NP vaccines as described above. Seven weeks later, all the mice were injected subcutaneously in the flank with B16. OVA melanoma cells $\left(1 \times 10^{5}\right)$ were acquired from $90 \%$ confluent cultures. The mice 
were examined every day and the tumor size was measured for every 2-3 days after appearance of first tumor using digital Vernier calipers. Mice with tumor sizes greater than $200 \mathrm{~mm}^{2}$ or meeting other predetermined humane endpoints were culled.

\section{Statistical Analysis}

Where applicable, results were analyzed for statistical significance with GraphPad Prism Version 6 using one-way analysis of variance (ANOVA) followed by Tukey's multiple comparison tests. Survival curves were compared using the Log-rank (Mantel-Cox) test.

\section{RESULTS}

\section{Formulation Characterization}

PLGA-NP containing the model antigen OVA and the adjuvants MPL and QA were successfully prepared by the double-emulsion method (Table I).

The sol-gel transition temperature and time were analyzed using oscillatory rheometry. A $17 \%(w / v)$ concentration of the polymer was chosen on the basis of preliminary studies (data not shown) examining the injectability of the hydrogels at room temperature. All the polymers analyzed were solutions at ambient temperature as indicated by the low complex viscosity values (Fig. 1a). With an increase in temperature, the thermoresponsive polymer solutions transformed into gels with a corresponding increase in complex viscosity. The sol-gel transition temperatures of $\mathrm{P} 1$ and $\mathrm{P} 2$ were determined to be 28 and $32^{\circ} \mathrm{C}$, respectively. The addition of PLGA-NP to the polymer solutions resulted in a decrease in the sol-gel transition temperature and increase in the complex viscosity of the gel (Fig. 1a). The gelation temperatures of P1 and P2 hydrogels containing PLGA-NP were 26 and $28^{\circ} \mathrm{C}$, respectively. All the pentablock copolymer formulations formed gels within $2 \mathrm{~min}$ at body temperature (Fig. 1b). The storage moduli (G') and loss moduli (G”) for the systems with or without nanoparticles at body temperature are depicted in supplementary Fig. 2. For all the formulations, $G^{\prime}$ is greater than $G$ ", indicating the formation of rigid gels.

\section{In Vitro Gel Stability and Release Studies}

Both pentablock copolymers were stable in aqueous environments, unlike the triblock copolymers (Fig. 2), and by the end of 30 days, only $43 \%$ of the P1 gel and $23 \%$ of $\mathrm{P} 2$ gel remained intact (Fig. 2). The dissolution of P1 gel was slightly slower than that of the $\mathrm{P} 2 \mathrm{gel}$ at all time points. The stability of the gels was increased in systems containing nanoparticles when compared to systems without nanoparticles. After 30 days, $65 \%$ of the $\mathrm{P} 1$ gel with nanoparticles and $57 \%$ of the P2 formulation with nanoparticles remained intact. NP stability in the hydrogels was confirmed by fluorescence microscopy (supplementary Fig. 3).

The in vitro release of FITC-OVA, MPL, and QA from PLGA-NP, thermoresponsive pentablock copolymer hydrogels, and PLGA-NP-loaded thermoresponsive hydrogels was evaluated over 30 days (Fig. 3). For the hydrophilic actives (FITC-OVA and QA), release was fastest and more complete from the NP ( $>85 \%$ release at 30 days). Release from the hydrogels was slower and less complete (60-70\% release at 30 days) and the inclusion of NP into the hydrogels further slowed release, with less than $40 \%$ of FITCOVA being released after 30 days. The inclusion of vaccineloaded NP into hydrogels also reduced the initial burst release of the hydrophilic actives. The release of the hydrophobic active MPL was less affected by the formulation; however, the same trends were present. Interestingly, release of actives from the $\mathrm{P} 1$ gels was slower than from the $\mathrm{P} 2$ gels.

\section{Pentablock Copolymer Hydrogels Induced Long-Lasting Antigen-Specific Immune Responses}

The cellular immune responses induced by triblock and pentablock copolymer hydrogels were investigated 21, 30, and 49 days after immunization (Fig. 4), and a number of interesting trends were apparent. Pentablock copolymer hydrogels induced significantly greater $\mathrm{CD}^{+} \mathrm{T}$ cell expansion than did the triblock hydrogels, the PLGA-NP and alum. Interestingly, the development of immune responses to the nanoparticle in hydrogel vaccines were delayed but after 49 days, P1 hydrogels containing nanoparticles stimulated a significantly greater expansion of $\mathrm{CD}^{+} \mathrm{T}$ cells than did the alum vaccine $(p<0.001)$. Mice immunized with P1 formulations had a higher number of $\mathrm{CD}^{+} \mathrm{T}$ cells compared to mice immunized with $\mathrm{P} 2$ formulations, both when loaded with soluble actives or with vaccine in PLGA-NP. The CD4 ${ }^{+}$ responses were not as clear cut but similar trends in responses were observed with the response to the PLGA-NP-loaded gels being delayed and the $\mathrm{P} 1$ vaccines being superior to the P2 vaccines. Mice immunized with a single dose of PLGA-NP or an alum-adjuvanted vaccine developed only weak $\mathrm{CD}^{+}$ responses.

Ova-specific IgG responses were determined by ELISA (Fig. 5). The alum-adjuvanted vaccines stimulated high antibody titres as expected while the triblock copolymer and the PLGA-NP vaccines stimulated modest antibody titres. In agreement with previous results, the pentablock copolymers containing soluble vaccines stimulated high antibody titres

Table I. Characterization of Blank PLGA-NP and FITC-OVA, MPL, and QA Loaded PLGA-NP. The Values Shown Are the Mean \pm $\mathrm{SD}(n=3)$

\begin{tabular}{|c|c|c|c|c|c|c|}
\hline Formulation & Z-Average (nm) & PDI & Zeta potential $(\mathrm{mV})$ & FITC-OVA EE (\%) & 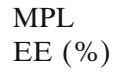 & $\begin{array}{l}\mathrm{QA} \\
\mathrm{EE}(\%)\end{array}$ \\
\hline Blank PLGA-NP & $250 \pm 12.9$ & $0.096 \pm 0.069$ & $-34.83 \pm 2.36$ & - & - & - \\
\hline FITC-OVA, MPL, QA PLGA-NP & $325 \pm 15.1$ & $0.257 \pm 0.039$ & $-42.42 \pm 3.59$ & $77.5 \pm 3.6$ & $65.1 \pm 3.1$ & $57.3 \pm 2.8$ \\
\hline
\end{tabular}




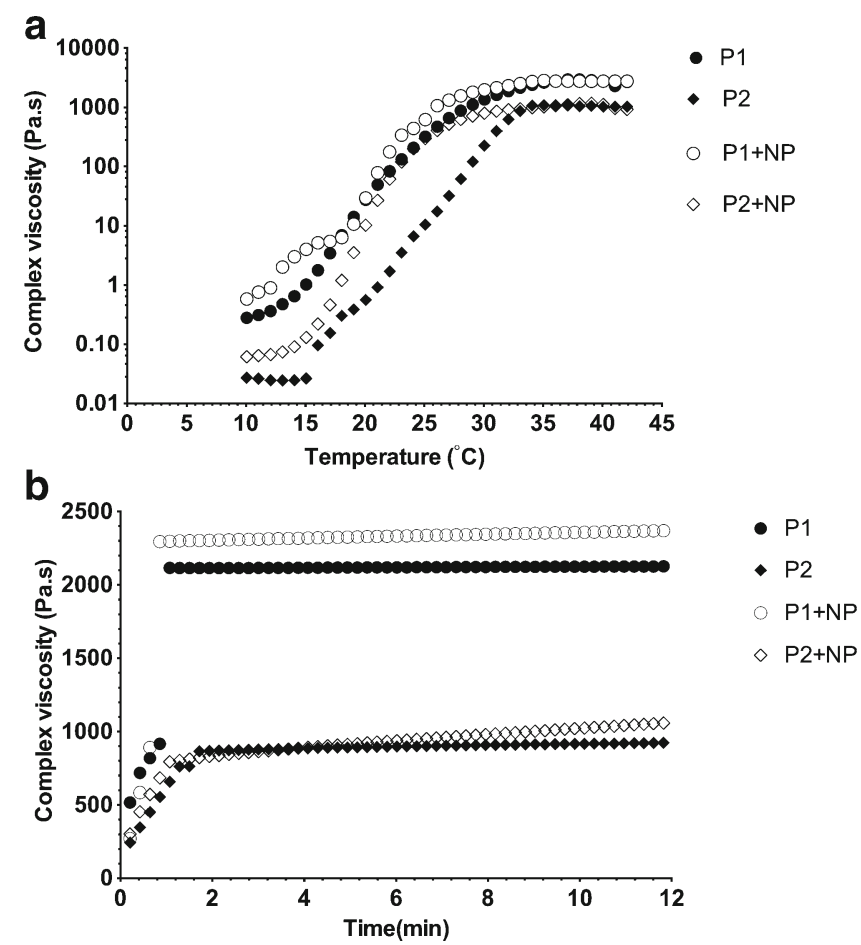

Fig. 1. a Sol-gel transition temperature and $\mathbf{b}$ sol-gel transition time at body temperature of $17 \%(w / v)$ pentablock copolymers in the presence or absence of PLGA-NP loaded with OVA, MPL, and QA

earlier than pentablock copolymers containing PLGA-NP vaccines. Antibody responses were long lived and detectable out to 49 days post immunization.

\section{Pentablock Copolymer Hydrogels Induced Long-Lived Cytotoxic Immune Responses}

The next step in evaluating the potential of these vaccine formulations was to use a functional assay. As these vaccines stimulated long-lasting cellular responses, the ability of the vaccines to stimulate memory cytotoxic immune responses able to prevent the establishment of tumors was investigated. From the previous experiments, the pentablock copolymer with the higher PCL to PLA ratio (P1) appeared to stimulate stronger immune responses than $\mathrm{P} 2$ and was therefore utilized in the tumor challenge experiment. Mice were

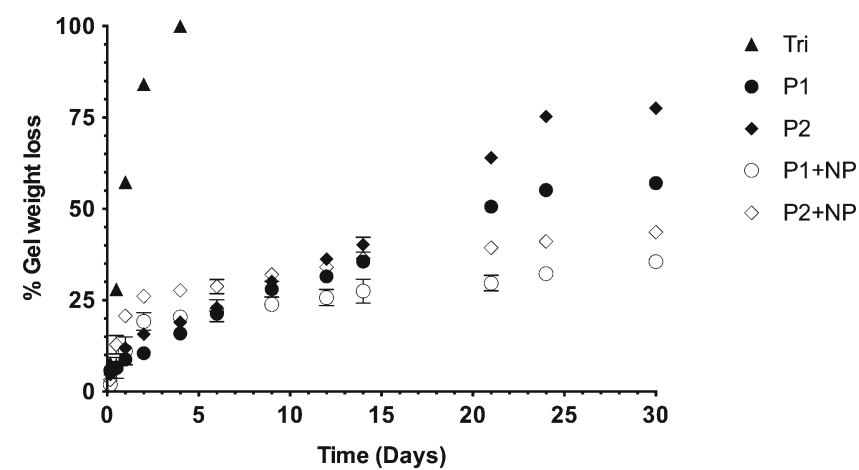

Fig. 2. In vitro gel stability of modified triblock copolymer (Tri) and pentablock copolymer hydrogels (P1 and P2) with and without PLGA-NP (NP) at $37^{\circ} \mathrm{C}$ in PBS buffer (pH 7.4). The values shown are the mean $\pm \mathrm{SD}(n=3)$
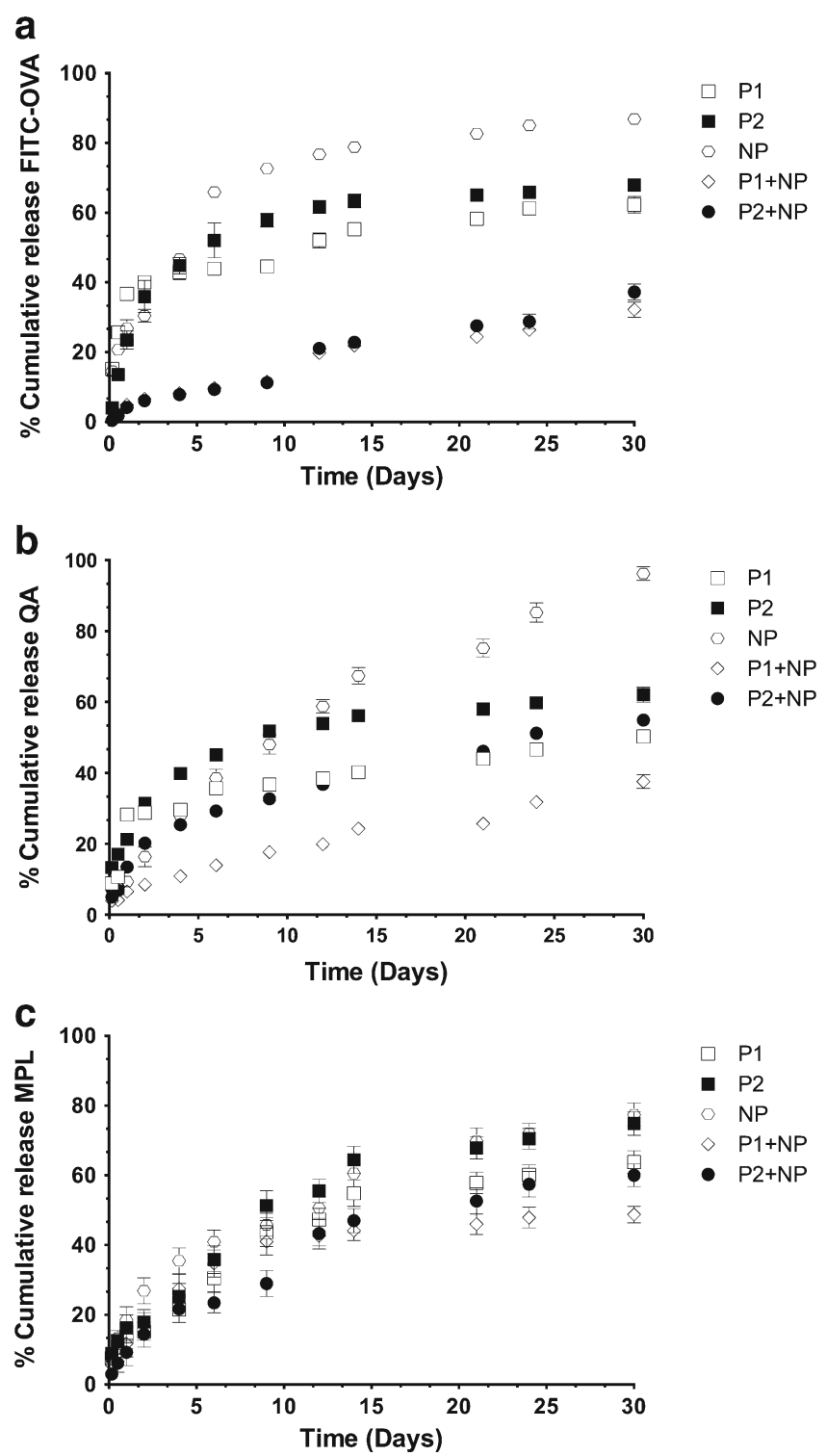

Fig. 3. In vitro release profiles of a FITC-OVA, $\mathbf{b} \mathrm{QA}$, and $\mathbf{c}$ MPL from PLGA-NP (NP) and pentablock copolymer hydrogels (P1 and P2) with and without PLGA-NP. Data shown are the mean $\pm \operatorname{SD}(n=3)$

immunized with P1 pentablock hydrogels loaded with either the soluble vaccine or the PLGA-NP vaccine or with a control vaccine with no antigen and 49 days later were injected subcutaneously with $1 \times 10^{5}$ B16.OVA melanoma cells (Fig. 6 and supplementary Fig. 4). Mice immunized with P1 hydrogels containing blank nanoparticles and the no treatment group had measureable tumors day 12 after tumor injection. After 25 days, $90 \%$ of the mice in the blank nanoparticle and no treatment group were euthanized after reaching predetermined clinical endpoints. After 40 days, $87 \%$ survival of mice immunized with the P1 hydrogel PLGA nanoparticle vaccine and $75 \%$ survival of mice immunized with the P1 hydrogels containing soluble vaccine were observed. The surviving mice were rechallenged on the other flank with $1 \times 10^{5}$ B16.OVA melanoma cells 60 days after the first challenge (109 days after immunization). After rechallenge, mice immunized with P1 
Day 21
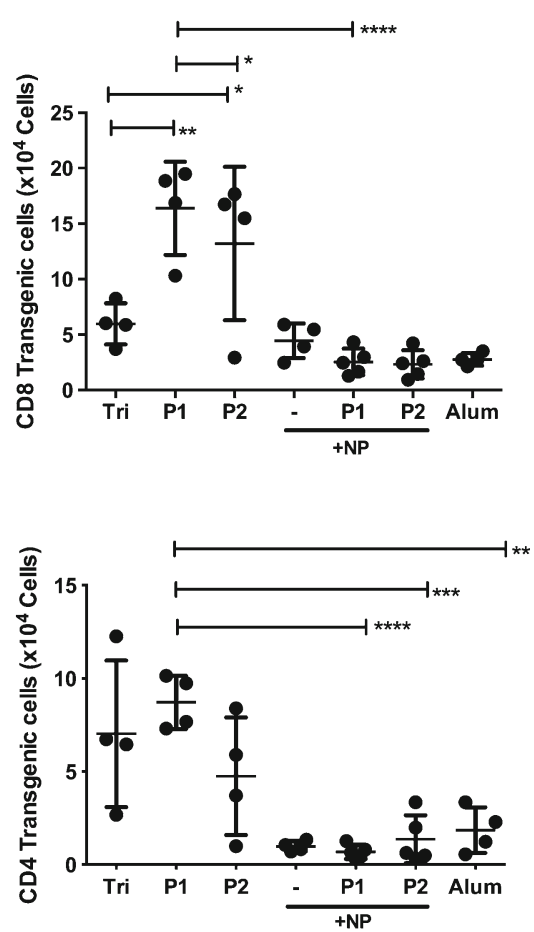

Day 30
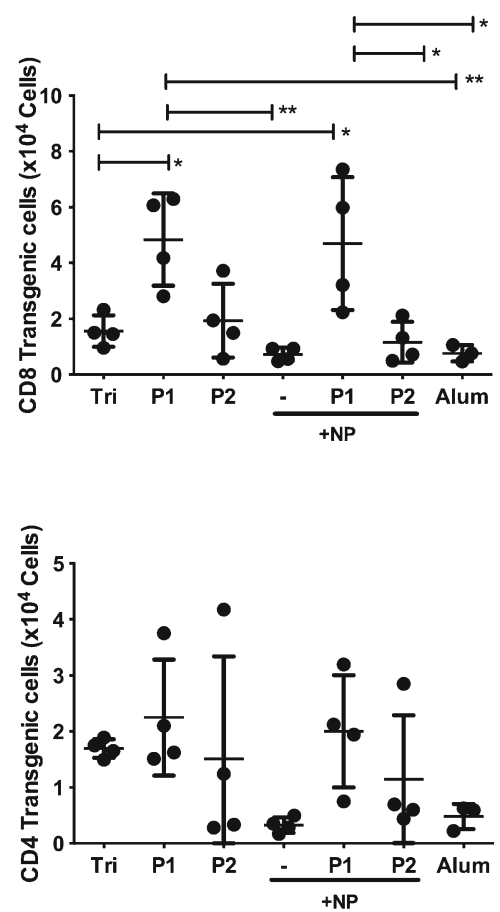

Day 49
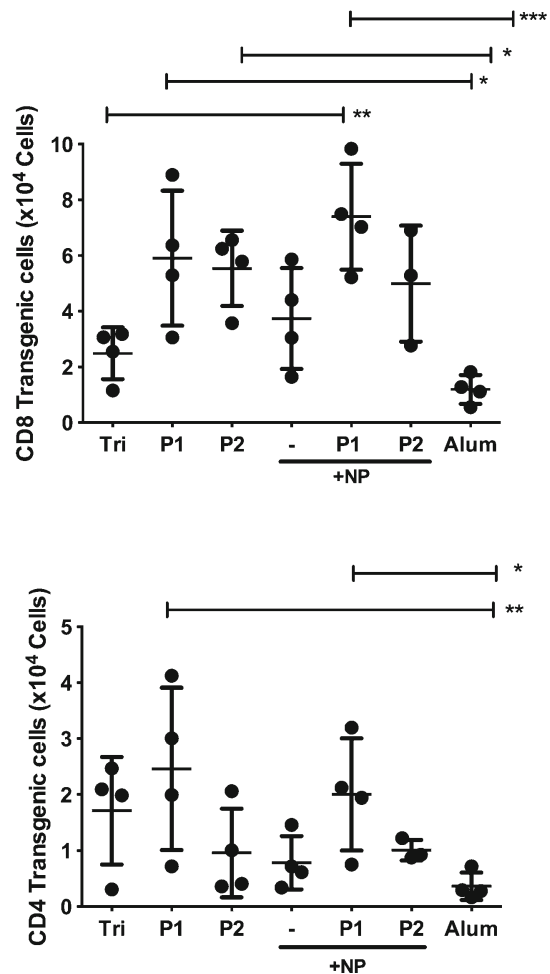

Fig. 4. Expansion of OVA-specific CD8 and CD4 transgenic T cells in lymph nodes 21, 30, and 49 days post immunization with the triblock copolymer (Tri), the pentablock copolymers (P1 and P2) with or without PLGA-NP (NP) or alum. Data presented are the individual mice and the group mean $\pm \operatorname{SD}(n=3-5$ mice, $* p \leq 0.05, * * p \leq 0.01, * * * p \leq 0.001, * * * * p \leq 0.0001)$

hydrogels with PLGA-NP vaccine had $75 \%$ survival, whereas $50 \%$ survival of mice immunized with the P1 hydrogels containing soluble vaccine was found. Mice immunized with the P1 hydrogels containing either soluble or NP vaccine had significantly greater survival than mice immunized with the P1 hydrogels containing blank NP $(p<0.0001)$. However, there was no significant difference in survival of mice immunized with the P1 hydrogels containing soluble or NP vaccine $(p=0.1611)$.

\section{DISCUSSION}

During a naturally occurring infection, antigens are released from the site of infection as the pathogen replicates until such time as the pathogens are destroyed by the immune system. This is the situation we wish to recreate with our vaccine formulation and the sustained release of antigens from depot formulations is one such strategy for achieving this (6). Modified triblock copolymer based thermoresponsive
Day 21

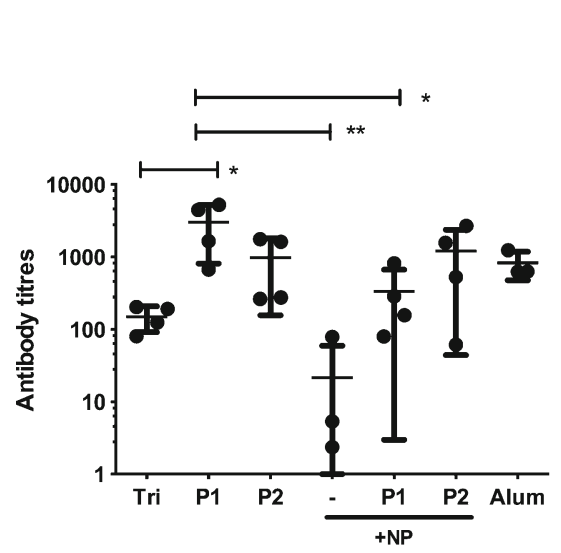

Day 30

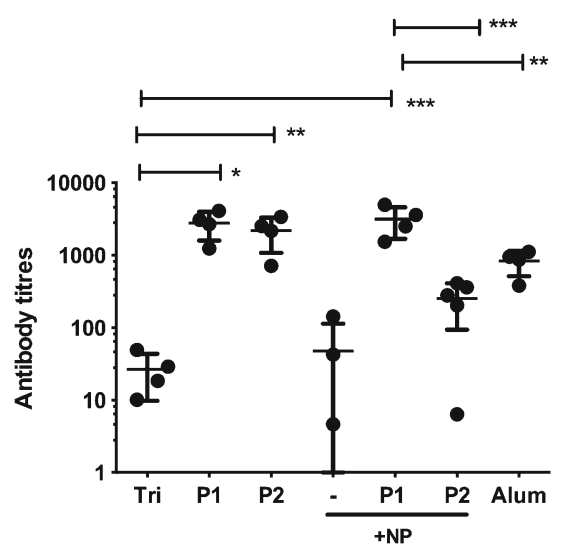

Day 49

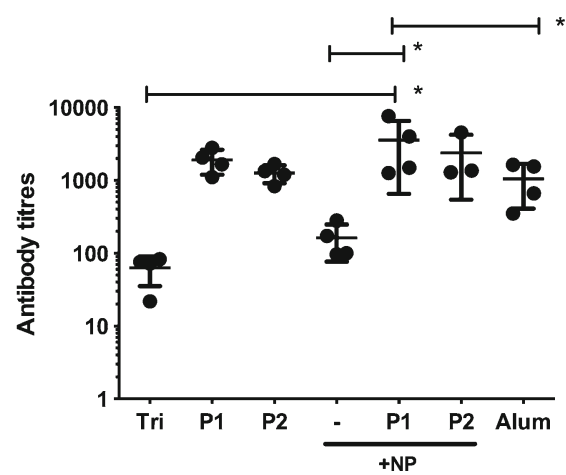

Fig. 5. Ova-specific $\operatorname{IgG}$ antibody titres in serum of mice after 21,30 , and 49 days post immunization with the triblock copolymer (Tri), the pentablock copolymers (P1 and P2) with or without PLGA-NP (NP) or alum. Data presented are the individual mice and the group mean \pm SD ( $n=3-5$ mice, $* p \leq 0.05, * * p \leq 0.01, * * * p \leq 0.001)$ 


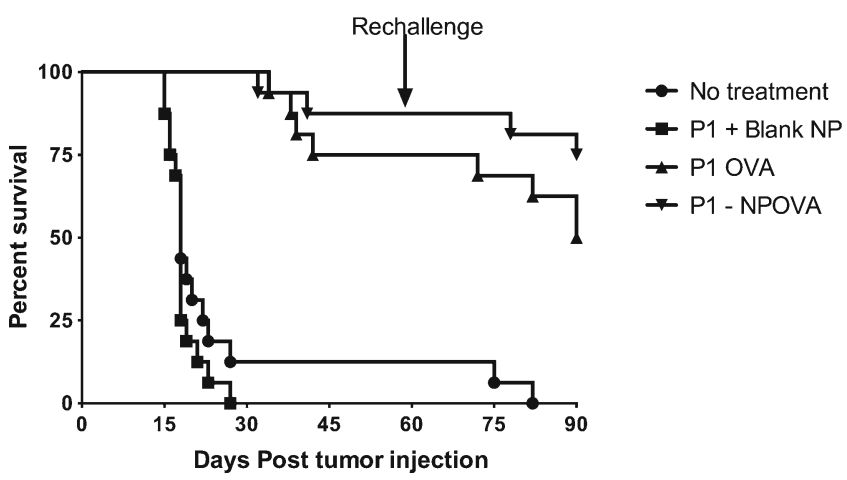

Fig. 6. Prophylactic anti-tumor response. Survival analysis of mice ( $n=16$, from two independent experiments) injected subcutaneously with $1 \times 10^{5}$ B16.OVA melanoma cells 49 days post immunization with the $\mathrm{P} 1$ pentablock gel loaded with blank PLGA-NP $(\mathrm{P} 1+$ blank NP), with vaccine-loaded PLGA-NP (P1-NPOVA) or with soluble vaccine (P1 OVA). Unimmunized mice were included as a control (no treatment). Surviving mice were rechallenged 60 days after the initial tumor challenge in the opposite flank with $1 \times 10^{5}$ B16.OVA melanoma cells

gels previously tested in our lab lacked stability (36); therefore in this study, we utilized pentablock copolymers. The two novel pentablock copolymers used in the study had the same block arrangement but differed in the ratio of PCL and PLA, having either a 3:1 (P1) or 1:1 (P2) ratio of PCL to PLA. The presence of PEG blocks made both polymers hydrophilic and able to be easily solubilized in aqueous systems. The aqueous polymer solutions consist of selfassembled polymeric micelles and upon an increase in temperature the micelles aggregate to form a gel (29). While both P1 and P2 transformed from solutions to gels at physiological temperature, $\mathrm{P} 1$ formed more rigid gels because of increased hydrophobic interactions between the micelles. The transition time of sol to gel for the polymers was less than 2 min, which is advantageous as it reduces the likelihood of dose dumping and the potential for the gel to be diluted with physiological fluids at the site of injection (39).

A particulate carrier loaded with the vaccine antigen and adjuvants was incorporated into the formulation in order to mimic the presence of pathogens and to facilitate synchronous uptake of the vaccine components by APCs $(7,8)$. PLGA-NP were chosen due to their biocompatibility, stability, and versatility. The addition of PLGA-NP to the gels decreased the sol-gel transition time and temperature, likely due to the increased hydrophobic interactions between the PLGA-NP and the gels.

A key requirement for the gels was stability and sustained release of antigen. Both gels were highly stable with only minor effects on stability resulting from the variation in the ratio of PCL to PLA. Increased hydrophobic interactions of PLGA-NP with the gels further increased the aqueous stability of particle-loaded formulations, consistent with the rheology measurements. The release of FITC-OVA, QA, and MPL from PLGA-NP exhibited an initial burst phase due to surface adsorption; however, when the nanoparticles were incorporated into the hydrogel, the burst effect was reduced due to the presence of the additional diffusion layer. The release of antigen and adjuvant from this combination formulation was overall slower as compared to formulations where antigen and adjuvant were loaded directly into the hydrogels, again due to the presence of two diffusion barriers - the particles and then the gel. The release of antigen and adjuvant in vitro was incomplete over the 30day examination period, and visual inspection of the mice culled at day 49 revealed the continued presence of a depot at the site of immunization. This type of release resembles a chronic rather than an acute infection, which may be detrimental to the immune response generated as chronic exposure to antigen can result in cell deletion or exhaustion (40). However, this will depend on how the antigen and adjuvant are being released over this extended period of time, for example if antigen continues to be released by itself after adjuvant release is complete or if release stays at high levels or reduces down to low levels. Low-level release of antigen in an immunogenic form may enrich memory cell populations (41) rather than exhausting effector cells.

The immunogenicity of these promising formulations was then examined. Immune responses to acute infections peak around day 10, therefore antigen release for at least 1015 days would be desirable for the stimulation of robust immune responses (42). Modified triblock copolymer hydrogels do not stimulate sustained immune responses due to poor stability and fast erosion of the gel (around 3 days) (36) and were included here as a control along with alum, a gold standard adjuvant widely used in the clinic. The pentablock copolymer hydrogels with their increased stability and sustained release of antigen and immunomodulators stimulated strong, long-lasting $\mathrm{T}$ cell and antibody responses. These results are in agreement with the recent investigations on chitosan thermoresponsive hydrogels as sustained vaccine delivery systems; however, those systems had less than optimal formulation characteristics $(36,43)$. Interestingly, the inclusion of PLGA-NP in the hydrogels delayed the induction of both cellular and humoral immune responses, possibly due to the reduced burst release and overall slower release of vaccine components. Recent literature suggests that multiple administrations of nanoparticle vaccines are required to stimulate effective cellular and humoral responses $(44,45)$. Consistent with these studies, our results demonstrated that a single dose of PLGA-NP vaccine is not sufficient to stimulate potent cellular or humoral immune responses.

The choice of adjuvant plays an important role in the formulation design of vaccines. Kamath et al. showed that the uptake of antigen by APCs before adjuvant reduces uptake and inhibits immunity, but that adjuvant uptake by APCs before antigen may not (7). Therefore, NPs were included in the formulation to ensure synchronous exposure of APC to both antigen and adjuvant. However, with the formulation used here, this did not seem necessary as the hydrogels loaded with the individual vaccine components also stimulated robust immune responses. This is likely related to the choice and combination of adjuvants used in this vaccine where a hydrophilic TLR-independent and a hydrophobic TLR-dependent adjuvant were used. The release of the adjuvants was similar or slightly faster than that of the protein antigen, which would result in APC being exposed to them either at the same time or to adjuvant before antigen, while the depot formulation would ensure exposure at the same place. The importance of the depot in creating a focus for the developing immune response was demonstrated by 
the much weaker immune responses stimulated by the NP vaccine.

P1 hydrogels were selected for the functional studies of immunity as these more rigid gels stimulated slightly stronger immune responses. As the vaccines stimulated strong $\mathrm{CD}^{+} \mathrm{T}$ cells responses, which are more difficult to generate as they require antigen cross presentation (46), a tumor challenge model was chosen to assess functional cytotoxicity (47). The tumor challenge was administered 49 days after vaccination, and remarkably a single dose of the hydrogel vaccine, with or without NP, was able to stimulate long-lived immunity able to mediate killing of the tumor cells in the majority of mice. Over half the mice were able to reject a further challenge 109 days after the initial immunization. These are impressive results for a single dose of a protein vaccine which are notorious for requiring booster immunizations and for not being strong stimulators of cytotoxic immune responses (48).

\section{CONCLUSION}

In this study, thermoresponsive injectable hydrogel vaccines were developed with the aim of delivering a subunit vaccine for a sustained period of time and to stimulate robust long-lived immune responses. This was achieved using two novel PEG-PCL-PLA-PCL-PEG pentablock copolymers. With the combination of adjuvants used here, the loading of the vaccine into a nanoparticle, to ensure synchronous uptake of both antigen and adjuvant by APC, was not required. However, this is likely a result of the physical and immunological properties of the adjuvants used and will not apply to all vaccines. Release of the vaccine in vitro from these formulations was incomplete over 30 days, which may be not optimal. Further studies should be carried out to accurately assess release in vivo and to develop a formulation which releases antigen over a shorter period of time (1015 days). These points notwithstanding, the vaccines developed here were able, after a single dose, to stimulate robust cellular and humoral immune responses able to reject tumor cells given a month and a half post immunization.

\section{ACKNOWLEDGMENTS}

SB is supported by University of Otago Postgraduate Scholarship.

\section{REFERENCES}

1. Arnon R, Ben-Yedidia T. Old and new vaccine approaches. Int Immunopharmacol. 2003;3(8):1195-204.

2. Kersten GFA, Crommelin DJA. Liposomes and ISCOMs. Vaccine. 2003;21(9-10):915-20.

3. Zinkernagel RM, Ehl S, Aichele P, Oehen S, Kundig T, Hengartner $\mathrm{H}$. Antigen localisation regulates immune responses in a dose- and time-dependent fashion: a geographical view of immune reactivity. Immunol Rev. 1997;156:199-209.

4. Myschik J, McBurney WT, Hennessy T, Phipps-Green A, Rades T, Hook S. Immunostimulatory biodegradable implants containing the adjuvant Quil-A-Part II: in vivo evaluation. J Drug Target. 2008;16(3):224-32.

5. Hailemichael Y, Dai Z, Jaffarzad N, Ye Y, Medina MA, Huang $\mathrm{X}-\mathrm{F}$, et al. Persistent antigen at vaccination sites induces tumor- specific CD8+ T cell sequestration, dysfunction and deletion. Nat Med. 2013;19(4):465-72.

6. Gordon S, Teichmann E, Young K, Finnie K, Rades T, Hook S. In vitro and in vivo investigation of thermosensitive chitosan hydrogels containing silica nanoparticles for vaccine delivery. Eur J Pharm Sci. 2010;41(2):360-8.

7. Kamath AT, Mastelic B, Christensen D, Rochat AF, Agger EM, Pinschewer DD, et al. Synchronization of dendritic cell activation and antigen exposure is required for the induction of Th1/Th17 responses. J Immunol. 2012;188(10):4828-37.

8. Foged C, Sundblad A, Hovgaard L. Targeting vaccines to dendritic cells. Pharm Res. 2002;19(3):229-38.

9. Gamvrellis A, Leong D, Hanley JC, Xiang SD, Mottram P, Plebanski M. Vaccines that facilitate antigen entry into dendritic cells. Immunol Cell Biol. 2004;82(5):506-16.

10. Baldridge JR, Crane RT. Monophosphoryl lipid A (MPL) formulations for the next generation of vaccines. Methods. 1999;19(1):103-7.

11. Baldridge JR, McGowan P, Evans JT, Cluff C, Mossman S, Johnson $\mathrm{D}$, et al. Taking a toll on human disease: toll-like receptor 4 agonists as vaccine adjuvants and monotherapeutic agents. Expert Opin Biol Ther. 2004;4(7):1129-38.

12. Kensil C, Wu J-Y, Soltysik S. Structural and immunological characterization of the vaccine adjuvant QS-21. In: Powell M, Newman M, editors. Vaccine design. Pharmaceutical biotechnology, vol. 6. US: Springer; 1995. p. 525-41.

13. Demana PH, Fehske C, White K, Rades T, Hook S. Effect of incorporation of the adjuvant Quil A on structure and immune stimulatory capacity of liposomes. Immunol Cell Biol. 2004;82(5):547-54.

14. Mikloska Z, Rückholdt M, Ghadiminejad I, Dunckley H, Denis M, Cunningham AL. Monophosphoryl lipid A and QS21 increase $\mathrm{CD} 8 \mathrm{~T}$ lymphocyte cytotoxicity to herpes simplex virus- 2 infected cell proteins 4 and 27 through IFN- $\gamma$ and IL-12 production. J Immunol. 2000;164(10):5167-76.

15. Rattanapak T, Young K, Rades T, Hook S. Comparative study of liposomes, transfersomes, ethosomes and cubosomes for transcutaneous immunisation: characterisation and in vitro skin penetration. J Pharm Pharmacol. 2012;64(11):1560-9.

16. Ruel-Gariepy E, Leroux JC. In situ-forming hydrogels-review of temperature-sensitive systems. Eur J Pharm Biopharm. 2004;58(2):409-26.

17. Jeong B, Kim SW, Bae YH. Thermosensitive sol-gel reversible hydrogels. Adv Drug Deliv Rev. 2002;54(1):37-51.

18. Dumortier G, Grossiord JL, Agnely F, Chaumeil JC. A review of poloxamer 407 pharmaceutical and pharmacological characteristics. Pharm Res. 2006;23(12):2709-28.

19. Kabanov AV, Batrakova EV, Alakhov VY. Pluronic block copolymers as novel polymer therapeutics for drug and gene delivery. J Control Release. 2002;82(2-3):189-212.

20. Kojarunchitt T, Hook S, Rizwan S, Rades T, Baldursdottir S. Development and characterisation of modified poloxamer 407 thermoresponsive depot systems containing cubosomes. Int $\mathrm{J}$ Pharm. 2011;408(1-2):20-6.

21. Paavola A, Yliruusi J, Rosenberg P. Controlled release and dura mater permeability of lidocaine and ibuprofen from injectable poloxamer-based gels. J Control Release. 1998;52(1-2):169-78.

22. Liu Y, Zhu Y-Y, Wei G, Lu W-Y. Effect of carrageenan on poloxamer-based in situ gel for vaginal use: improved in vitro and in vivo sustained-release properties. Eur J Pharm Sci. 2009;37(3-4):306-12.

23. El-Kamel AH. In vitro and in vivo evaluation of Pluronic F127based ocular delivery system for timolol maleate. Int J Pharm. 2002;241(1):47-55.

24. Gong C, Shi S, Dong P, Kan B, Gou M, Wang X, et al. Synthesis and characterization of PEG-PCL-PEG thermosensitive hydrogel. Int J Pharm. 2009;365(1-2):89-99.

25. Ma G, Miao B, Song C. Thermosensitive PCL-PEG-PCL hydrogels: synthesis, characterization, and delivery of proteins. J Appl Polym Sci. 2010;116(4):1985-93.

26. Gou M, Zheng L, Peng X, Men K, Zheng X, Zeng S, et al. Poly(epsilon-caprolactone)-poly(ethylene glycol)-poly(epsiloncaprolactone) (PCL-PEG-PCL) nanoparticles for honokiol delivery in vitro. Int J Pharm. 2009;375(1-2):170-6. 
27. Chen CC, Chueh JY, Tseng H, Huang HM, Lee SY. Preparation and characterization of biodegradable PLA polymeric blends. Biomaterials. 2003;24(7):1167-73.

28. Ge H, Hu Y, Yang S, Jiang X, Yang C. Preparation, characterization, and drug release behaviors of drug-loaded $\varepsilon$ caprolactone/L-lactide copolymer nanoparticles. J Appl Polym Sci. 2000;75(7):874-82.

29. Patel SP, Vaishya R, Mishra GP, Tamboli V, Pal D, Mitra AK. Tailor-made pentablock copolymer based formulation for sustained ocular delivery of protein therapeutics. J Drug Deliv. 2014;2014:401747. doi:10.1155/2014/401747.

30. Silva JM, Videira M, Gaspar R, Preat V, Florindo HF. Immune system targeting by biodegradable nanoparticles for cancer vaccines. J Control Release. 2013;168(2):179-99.

31. Hamdy S, Haddadi A, Somayaji V, Ruan D, Samuel J. Pharmaceutical analysis of synthetic lipid A-based vaccine adjuvants in poly (D, L-lactic-co-glycolic acid) nanoparticle formulations. J Pharm Biomed. 2007;44(4):914-23.

32. Könnings S, Copland MJ, Davies NM, Rades T. A method for the incorporation of ovalbumin into immune stimulating complexes prepared by the hydration method. Int J Pharm. 2002;241(2):385-9.

33. Bobbala S, McDowell A, Hook S. Quantitation of the immunological adjuvants, monophosphoryl lipid A and Quil A in poly (lactic-co-glycolic acid) nanoparticles using high performance liquid chromatography with evaporative light scattering detection. J Chromatogr B. 2015;975:45-51.

34. Barnden MJ, Allison J, Heath WR, Carbone FR. Defective TCR expression in transgenic mice constructed using cDNA-based aand b-chain genes under the control of heterologous regulatory elements. Immunol Cell Biol. 1998;76(1):34-40.

35. Hogquist KA, Jameson SC, Heath WR, Howard JL, Bevan MJ, Carbone FR. T cell receptor antagonist peptides induce positive selection. Cell. 1994;76(1):17-27.

36. Kojarunchitt T, Baldursdottir S, Dong Y-D, Boyd BJ, Rades T, Hook S. Modified thermoresponsive poloxamer 407 and chitosan sol-gels as potential sustained-release vaccine delivery systems. Eur J Pharm Biopharm. 2015;89:74-81.

37. Filatenkov AA, Jacovetty EL, Fischer UB, Curtsinger JM, Mescher MF, Ingulli E. CD4 T cell-dependent conditioning of dendritic cells to produce IL-12 results in CD8-mediated graft rejection and avoidance of tolerance. J Immunol. 2005;174(11):6909-17.

38. McBurney WT, Lendemans DG, Myschik J, Hennessy T, Rades T, Hook S. In vivo activity of cationic immune stimulating complexes (PLUSCOMs). Vaccine. 2008;26(35):4549-56.

39. Charrueau C, Tuleu C, Astre V, Grossiord JL, Chaumeil JC. Poloxamer 407 as a thermogelling and adhesive polymer for rectal administration of short-chain fatty acids. Drug Dev Ind Pharm. 2001;27(4):351-7.

40. Mueller SN, Ahmed R. High antigen levels are the cause of T cell exhaustion during chronic viral infection. Proc Natl Acad Sci U S A. 2009;106(21):8623-8.

41. Kim TS, Hufford MM, Sun J, Fu Y-X, Braciale TJ. Antigen persistence and the control of local $\mathrm{T}$ cell memory by migrant respiratory dendritic cells after acute virus infection. J Exp Med. 2010;207(6):1161-72.

42. Whitmire JK, Murali-Krishna K, Altman J, Ahmed R. Antiviral CD4 and CD8 T-cell memory: differences in the size of the response and activation requirements. Philos Trans R Soc Lond B Biol Sci. 2000;355(1395):373-9.

43. Highton AJ, Kojarunchitt T, Girardin A, Hook S, Kemp RA. Chitosan hydrogel vaccine generates protective CD8 T cell memory against mouse melanoma. Immunol Cell Biol. 2015;93(7):634-40.

44. Varypataki E, van der Maaden K, Bouwstra J, Ossendorp F, Jiskoot W. Cationic liposomes loaded with a synthetic long peptide and poly(I:C): a defined adjuvanted vaccine for induction of antigen-specific $\mathrm{T}$ cell cytotoxicity. AAPS J. 2015;17(1):216-26.

45. Joshi V, Geary S, Salem A. Biodegradable particles as vaccine delivery systems: size matters. AAPS J. 2013;15(1):85-94.

46. Joffre OP, Segura E, Savina A, Amigorena S. Cross-presentation by dendritic cells. Nat Rev Immunol. 2012;12(8):557-69.

47. Gilboa E. The promise of cancer vaccines. Nat Rev Cancer. 2004;4(5):401-11.

48. Atanackovic D, Altorki NK, Cao Y, Ritter E, Ferrara CA, Ritter $\mathrm{G}$, et al. Booster vaccination of cancer patients with MAGE-A3 protein reveals long-term immunological memory or tolerance depending on priming. Proc Natl Acad Sci U S A. 2008;105(5):1650-5. 